\title{
NOTES ON THE BERTINI INVOLUTION
}

\author{
ETHEL I. MOODY 1
}

1. Introduction. Given a pencil of plane cubic curves

$$
\lambda w(x)+\mu w^{\prime}(x)=0
$$

with the vertices of the reference triangle among its base points. Arranged as to $(0,0,1)$ the equations may be written

with

$$
\begin{gathered}
w(x)=\stackrel{2}{x_{3} u_{1}}+x_{3} u_{2}+u_{3}, \\
w^{\prime}(x)=\stackrel{2}{2} u_{1}^{\prime}+x_{3} u_{2}^{\prime}+u_{3}^{\prime},
\end{gathered}
$$

$$
\begin{aligned}
& u_{1}=a_{1} x_{1}+a_{2} x_{2}, \quad u_{1}^{\prime}=a_{1}^{\prime} x_{1}+a_{2}^{\prime} x_{2}, \\
& u_{2}=b_{1} x_{1}^{2}+b_{2} x_{1} x_{2}+b_{3} x_{2}^{2}, \quad u_{2}^{\prime}, \\
& u_{3}=c_{1} x_{1}^{2} x_{2}+c_{2} x_{1} x_{2}^{2}, \quad u_{3}^{\prime},
\end{aligned}
$$

and $a_{i}, a_{i}^{\prime}, b_{i}, b_{i}^{\prime}, c_{i}, c_{i}^{\prime}$ generic constants.

A point $y$ of the plane fixes the curve of the pencil (1) passing through it, hence

$$
w(x) w^{\prime}(y)-w^{\prime}(x) w(y)=0,
$$

which may be written in the form

$$
\begin{aligned}
W_{3}(x)= & x_{3}\left(A_{1} x_{1}+A_{2} x_{2}\right)+x_{3}\left(B_{1} x_{1}^{2}+B_{2} x_{1} x_{2}+B_{3} x_{2}^{2}\right) \\
& +C_{1} x_{1}^{2} x_{2}+C_{2} x_{1} x_{2}^{2}=0
\end{aligned}
$$

in which $A_{i}=a_{i} w^{\prime}(y)-a_{i}^{\prime} w(y)$, and similarly for $B_{i}$ and $C_{i}$. The tangent to $W_{3}(x)=0$ at $(0,0,1)$ is

$$
A_{1} x_{1}+A_{2} x_{2}=0 \text {, }
$$

which meets the curve again at $R=\left(r_{1}, r_{2}, r_{3}\right)$,

Received by the editors August 31, 1942.

${ }^{1}$ Miss Moody, Ph.D. Cornell University, an instructor in mathematics at Pennsylvania State College, was killed in an automobile accident April 11, 1941. I had suggested that she compare my cumbersome method of derivation of the equations of this transformation (Amer. J. Math. vol. 33 (1911) pp. 327-336) with that of employing a pencil of cubic curves. The following notes were found among her posthumous papers sent me recently. The equations of the Bertini involution are simpler than those previously known, and other properties found may be extended by others.

VirgIL SNyder 


$$
\rho r_{1}=A_{2} r_{1}^{\prime}, \quad \rho r_{2}=-A_{1} r_{2}^{\prime}, \quad \rho r_{3}=A_{1} A_{2} r_{3}^{\prime},
$$

wherein

$$
r_{1}^{\prime}=B_{1} A_{2}^{2}-B_{2} A_{1} A_{2}+B_{3} A_{1}^{2}, \quad r_{3}^{\prime}=A_{2} C_{1}-A_{1} C_{2} .
$$

As $\lambda: \mu$ describes the pencil (3), the locus of $R$ is

$$
\gamma_{4}(x)=x_{1} A_{1}+x_{2} A_{2}=0,
$$

a quartic curve having a triple point at $(0,0,1)$ and passing simply through the remaining base points. It is completely fixed by these conditions.

2. The Bertini involution. The locus $R$ meets every curve of the pencil (3) in one point apart from base points. The line $R y$ meets $W_{3}(x)=0$ in a third point $y^{\prime}$. It is proposed to study the involutorial transformation $y \sim y^{\prime}$.

The point $y^{\prime}$ is associated with the points $y$ and $R$ by equations of the form $y^{\prime}=l y+m r$.

Substitute $y^{\prime}$ for $x$ in $W_{3}(x)=0$. Since $W_{3}(y)=0$ and $W_{3}(r)=0$, the equation in $l: m$ is linear. After various manipulations, it is found that $\gamma_{4}(y)$ appears as a cubic factor, and the equations of the transformation may be written in the form

$$
\begin{aligned}
& y_{1}^{\prime}=\phi_{6}(y)\left[A_{2}^{2} \phi_{6}(y)+B_{3} r_{1}^{\prime}\right]_{y_{1}}, \\
& y_{2}^{\prime}=\psi_{6}(y)\left[A_{1}^{2} \psi_{6}(y)+B_{1} r_{1}^{\prime}\right]_{y_{2}}, \\
& y_{3}^{\prime}=\psi_{6}(y) \phi_{6}(y) C_{5} .
\end{aligned}
$$

A subscript applied to a bracket indicates that the quantity enclosed has the subscript as a factor and that the factor has been removed.

$$
\begin{aligned}
C_{5}(y)= & A_{2}\left[B_{1}+\left(a_{1} b_{1}^{\prime}-a_{1}^{\prime} b_{1}\right) y_{1} y_{2}^{2}\right]_{y_{2}} \\
& +\left[A_{1}-\left(a_{1}^{\prime} b_{1}-a_{1} b_{1}^{\prime}\right) \cdot y_{2}^{2} y_{3}\right]_{y_{2}}\left[A_{2} y_{3}+B_{3} y_{2}\right]_{y_{1}} \\
& +\left(a_{1} b_{1}^{\prime}-a_{1} b_{1}^{\prime}\right) B_{3} y_{1} y_{3}, \\
\phi_{6}(y)= & A_{1} C_{2}+y_{3} C_{5}(y), \\
\psi_{6}(y)= & A_{2} C_{1}+y_{3} C_{5}(y) .
\end{aligned}
$$

These expressions have no common factor and are all in terms of the coefficients in $W_{3}(y)$. From the forms of the equations it follows at once that the transformation is of order 17 . The point $(0,0,1)$ is not a fundamental point. It is a fixed point of the transformation. The other base points of the pencil enter symmetrically. $\psi_{6}(y)=0$ is the principal curve for $(1,0,0)$ and $\phi_{6}(y)=0$ for $(0,1,0)$. 
From the forms of the equations of these principal curves it is seen that each has a triple point at the fundamental point to which it belongs, and a double point at each of the other seven. There are no other fundamental points, hence

$$
C_{1} \sim C_{17}: 8 P_{i}^{6} \quad \quad P_{i} \sim p_{6}: P_{i}^{3} 7 P_{k}^{2}, \quad k \neq i .
$$

It is the general Bertini involution.

3. Fixed points of $B_{17}$. From the method of defining $y^{\prime}$ it follows that every cubic curve of the pencil is transformed itself from the central involution from its associated point $R$.

From $R$ can be drawn four tangents to $W_{3}(y)=0$ apart from the one at $R$. But one of these touches the curve at $(0,0,1)$, as $R$ describes $y(x)=0$. The locus of the other points of tangency is obtained by eliminating $\lambda: \mu$ from (1) and the polar conic of $R$ as to $W_{3}(y)=0$. By using the same procedure to affect reductions, the equation of the locus is found to be

$$
K(x)=\phi_{6}(y)\left[A_{1} y_{3}+B_{1} y_{1}\right]_{y_{2}}-\psi_{6}(y)\left[A_{3} y_{3}+B_{3} y_{2}\right]_{y_{1}}=0 .
$$

It is of order 9 , has each base point of $\left|W_{3}(y)\right|$ except $(0,0,1)$ to multiplicity 3 , and no other multiple points. It is of genus 4 . Although $(0,0,1)$ is a fixed point, its coordinates do not satisfy $K(x)=0 .^{2}$

It is at once confirmed that at the points $(1,0,0)$ and $(0,1,0)$ the curve $K(x)=0$ has the same tangents as $\psi_{6}(y)=0, \phi_{6}(y)=0$, respectively. Since these points have no particular role in the determining equations, we infer that $K(y)=0$ has the same tangents at each base point as the principal curve of that point.

4. Particular pencils. Suppose all the curves of the pencil (1) have a common tangent at $(0,0,1)$. This requires that a second base point approaches $(0,0,1)$ in the direction of the fixed tangent. The curve $\gamma_{4}(x)=0$ now consists of the fixed tangent and the cubic curve of the pencil having a double point at $(0,0,1)$. The transformation reduces to the general Geiser involution having the other seven base points of the pencil as fundamental points.

$$
\begin{gathered}
C_{1} \sim C_{8}: 7 P_{i}^{2}, \quad \sim p_{3}: P_{i}^{2} 6 P_{k}, \quad k \neq i, \\
K_{6}: 7 P_{i}^{2} .
\end{gathered}
$$

Conversely, given any seven points in the plane: if the Geiser involution be determined and its points of invariant points be con-

${ }^{2}$ In Hudson, Cremona transformations, p. 127 , it is erroneously stated that $(0,0,1)$ is triple on $K(x)=0$. 
structed, a tangent to $K=0$ has the role of the remaining (adjacent) base points of the pencil of cubics belonging to the point on $K$.

Further particularizations of the Bertini involution are thus reduced to special cases of the Geiser type. ${ }^{3}$

5. Product of two Bertini involutions. Returning to the general case, consider the sequences of two Bertini involutions, first $T_{3}$ with the base point $(0,0,1)$ of the pencil, followed by $T_{2}$ with $(0,1,0)$ as the special vertex.

By $T_{3}$

By $T_{2}$

$$
C_{1} \sim C_{17}: 8 P_{i}^{6}, \quad i \neq 3 .
$$

$$
C_{17}: 8 P_{i}^{6} \sim C_{37}: P_{3}^{18} P_{2}^{6} 7 P_{i}^{2}, \quad i \neq 2,3 .
$$

This transformation is not periodic. Its inverse is the product of the same components taken in inverse order.

6. Components of the curve of fixed points of $T_{3} T_{2}$ and $T_{2} T_{3}$. Consider the $(x)=0$ belonging to $T_{3}$ and that belonging to $T_{2}$. These two curves meet in three points $Q_{i}$ apart from base points. Since from $Q_{i}$ the operations $T_{3}$ and $T_{2}$ are identical, it follows that the product of the two involutions leaves every point of the cubic of the pencil determined by $Q_{i}$ fixed, hence:

The product of $T_{2}$ and $T_{3}$ leaves three cubics of the pencil point by point invariant.

7. Product of three $T_{i}$. By direct application of the formula we obtain $T_{3} T_{2} T_{1}$

$$
\begin{aligned}
& C_{1} \sim C_{53}: P_{1}^{12} P_{2}^{24} P_{3}^{12} 6 P_{i}^{18}, \quad i \neq 1,2,3 \text {, } \\
& P_{1} \sim p_{12}: P_{1}^{3} P_{2}^{6} P_{3}^{2} 6 P_{i}^{4} \text {, } \\
& P_{2} \sim p_{24}: P_{1}^{6} P_{2}^{11} P_{3}^{6} 6 P_{i}^{8} \text {, } \\
& P_{3} \sim p_{12}: P_{1}^{2} P_{2}^{6} P_{3}^{3} 6 P_{i}^{4} \text {, } \\
& P_{i} \sim p_{18}: P_{1}^{4} P_{2}^{8} P_{3}^{4} P_{i}^{7} 5 P_{j}^{6} \quad j \neq 1,2,3, i .
\end{aligned}
$$

If $T_{3} T_{2} T_{1}$ be calculated, its characteristic and that of its inverse are found to be identical hence:

The product of three different Bertini involutions associated with the same pencil of cubic curves is involutorial.

Pennsylvania State College

${ }^{3} \mathrm{~V}$. Snyder, Conjugate line congruences contained in a bundle of quadric surfaces, Trans. Amer. Math. Soc. vol. 11 (1911) pp. 371-387. 